\title{
Indole: An evolutionarily conserved influencer of behavior across kingdoms
}

Jeffery K. Tomberlin ${ }^{1)^{*}}$, Tawni L. Crippen ${ }^{2)}$, Guoyao $\mathrm{Wu}^{3)}$, Ashleigh S. Griffin ${ }^{4)}$, Thomas K. Wood $^{5)}$ and Rebecca M. Kilner ${ }^{6)}$

${ }^{1)}$ Department of Entomology, Texas A\&M University, College Station, TX, USA

2) Southern Plains Agricultural Research Center, Agricultural Research Service, U.S. Department of Agriculture, College Station, TX, USA

3) Department of Animal Science, Texas A\&M University, College Station, TX, USA

4) Department of Zoology, University of Oxford, Oxford, UK

5) Department of Chemical Engineering \& Biochemistry and Molecular Biology, Pennsylvania State University, University Park, PA, USA

6) Department of Zoology, University of Cambridge, Cambridge, UK

\section{*Corresponding author:}

Jeffery K. Tomberlin

E-mail: jktomberlin@tamu.edu

Keywords: behavioral cue; behavioral signal; inter-kingdom interactions; competition; mutualism

Abbreviations: QS, quorum-sensing; Trp, tryptophan. 
Abstract. Indole is a key environmental cue that is used by many organisms. Based on its biochemistry, we suggest indole is used so universally, and by such different organisms, because it derives from the metabolism of tryptophan, a resource essential for many species yet rare in nature. These properties make it a valuable, environmental cue for resources almost universally important for promoting fitness. We then describe how indole is used to coordinate actions within organisms, to influence the behavior of conspecifics and can even be used to change the behavior of species that belong to other kingdoms. Drawing on the evolutionary framework that has been developed for understanding animal communication, we show how this is diversely achieved by indole acting as a cue, a manipulative signal, and an honest signal, as well as how indole can be used synergistically to amplify information conveyed by other molecules. Clarifying these distinct functions of indole identifies patterns that transcend different kingdoms of organisms. 


\section{Introduction}

With the advent of high-throughput sequencing and other molecular techniques, researchers are now able to peer into the microscopic world and determine the ecological and evolutionary interactions of single cell organisms in more detail than ever before, yielding new insights into the way in which microbial cells interact with each other and with other organisms such as plants and animals. Recent reviews have highlighted that microbes, and particularly bacteria, are adept at influencing the behavior of animals [1-3]. Furthermore, researchers from across a multitude of disciplines have discovered a number of molecules produced by microbes that mediate changes in animal behavior. However, one molecule in particular, indole, seems to be ubiquitous in nature across the organismal scale from microbes and plants to invertebrates and vertebrates [4,5] (Table 1). The goal of this review is to describe the diverse ways in which indole mediates interactions between organisms, and to map the extraordinary natural history that has recently been uncovered onto longestablished evolutionary concepts from the study of animal communication [6]. The purpose is to understand more about the evolution and function of indole in the natural world, and to identify gaps in understanding that might be profitably filled by future research.

The initial portion of the review covers the biochemistry of indole, to identify special properties that might explain its ubiquity in mediating interactions among organisms. We then review the types of interactions between organisms that are regulated by indole, and attempt to classify them using existing concepts from the theory of animal communication. To make sense of what follows, we therefore begin with a brief primer in the terminology and concepts from evolutionary communication theory. This lays the foundation for our understanding of the function of indole in 
mediating interactions within and among kingdoms.

\section{Setting the stage- a signaling primer}

Evolutionary theory of animal communication makes important distinctions between the different ways that animals can collect information from one another, and their wider world. The animal collecting information is often referred to as the receiver. In the simplest case, receivers can use the behavior of others as a source of information. For example, bees (Hymenoptera: Apidae) are able to gain information about the nectar content of flowers on a plant simply by the presence of other bees - the presence of conspecifics is a "cue" to the potential availability of a resource. Importantly, the cue conveys useful information to the receiver but it has not evolved specifically for that purpose. Honeybees, Apis sp., (Hymenoptera: Apidae) also have famously sophisticated ways of actively transmitting information about the location of nectar-producing flowers to one another through the waggle dance. In this case, the location of a resource is being "signaled" and the individual imparting that information is known as the signaler. The key distinction between a signal and a cue is that the signal has evolved for the purpose of imparting information, because the signaler benefits in some way as a result of the receiver acting on the information conveyed.

An animal will only respond to a cue if to do so is of benefit to that receiver otherwise it will be selected to ignore it. An animal that responds to a signal could benefit too, if the information sent by the signaler is accurate and useful to the receiver - in other words, if it is an 'honest signal'. The conditions that enforce the evolution of honest signaling are still the subject of some debate [6], but one suggestion is that honesty evolves when the nature of the signal is intimately 
connected to the information it conveys, making it harder to fake. The depth of a toad's croak is tightly associated with its body size, for example, and so accurately conveys the competitive ability of the sender [7].

However, signals need not be honest, and signalers can potentially use their signals to manipulate others [8]. A manipulative signal is one, which brings a fitness benefit to the signaler, but at some fitness cost to the receiver. Any receiver that is routinely manipulated in this way is then placed under intense selection to ignore the manipulative signal, because it will instantly gain fitness as a result. Nevertheless, some receivers remain vulnerable to manipulation by signalers. They may be caught in a sensory trap, for example. This could happen when it is sometimes - though not always - adaptive for receivers to respond to a particular signal, and the receiver cannot distinguish the contexts when it should and should not respond. Manipulative signalers can further exploit receiver uncertainty here by sending signals that mimic the credible signal to which the signaler is attuned. This is how cuckoo nestlings succeed in manipulating their host parents into feeding them, for example [7].

Finally, whether or not a signal is honest, it is under selection to be salient and detectable by the receiver. Signals therefore often comprise multiple elements [9]. More than one element might convey the same information, and this redundancy of information might ensure that the message gets across even if it is partially degraded during transmission [10]. Other elements might convey no information at all, but serve simply to amplify information conveyed by other elements of the display [11]. Or each element of the display might convey different information, transmitting multiple messages simultaneously to the receiver [10]. The different parts of a nestling's begging display probably serve each of these functions, for example $[12,13]$ 


\section{Indole biochemistry and production}

We now return our attention specifically to indole and apply these concepts to understanding its many functions in the natural world. Since its initial discovery in the mid-twentieth century, a tremendous amount has been determined about indole's chemistry, relevance in ecology, and more recently its inter-kingdom interactions. A key point is that indole is a by-product from the metabolism of tryptophan (Ltryptophan (Trp; $\alpha$-amino- $\beta$-3-indolepropionic acid)), a large neutral amino acid containing an aromatic ring [14]. Trp is a relatively rare but nutritionally essential (indispensable) amino acid for mammals and it is generally more abundant in animalthan in plant-source foods. Importantly, Trp can be degraded by free-living bacteria, as well as plants and bacterial flora in animals to yield indole or indole-based compounds. In monogastric animals (e.g. pigs and rats), $\sim 15 \%$ of dietary Trp is degraded by intestinal bacteria, while in animal cells, three pathways are responsible for degrading Trp in a highly cell- and tissue-specific manner: the kynurenine, serotonin, and transamination pathways [15]; however, these paths do not produce indole from tryptophan, so animals acquire indole from the bacteria which colonize them.

The biochemistry of indole provides some clues to explain its ubiquity in mediating interactions among organisms in nature. First, as previously mentioned, it can be readily produced by plants, by bacteria in animals and by free-living microbes simply through the metabolism of tryptophan. This means that in principle any of these organisms can produce indole. Second, tryptophan is a rare and valuable resource, and the production of indole, its metabolite, provides useful information about its potential location. In other words, the rarity yet importance of tryptophan makes indole a valuable cue for diverse organisms. Accordingly, cells are able to detect and respond to Trp metabolites such as indole and can quickly change their 
patterns of gene expression as a result. This is well illustrated by detailed analyses of the action of indole (and other Trp metabolites) in animal cells (Figure 1). Here Trp metabolites are natural ligands and activators of the aryl hydrocarbon receptor (AhR; also known as dioxin receptor) [16], which is a cytosolic ligand-activated transcription factor. AhR is normally present in a dormant state but upon ligand binding, AhR undergoes a conformational change leading to the exposure of a nuclear localization signal. Thereafter, the ligand-activated AhR translocates into the nucleus, dissociates from the complex, and forms a heterodimer with the closely related Arnt protein in the nucleus. This in turn enhances expression of target genes.

A final key point is that indole closely resembles human and plant hormones such as serotonin and indole-3-acetic acid, respectively. This has also led to speculation that indole is the archetype for cell hormones [17]. It might also explain how indole can mediate interactions among kingdoms, ranging from bacteria stimulating seed germination in orchids [18], microalga [19] and diatom division [20] to fungi causing wilt in chickpeas [21], and increasing chlorophyll content and root growth in rice [22]. In short, the biochemistry of indole explains why it is a valuable cue for many diverse organisms and shows how cells are organized to detect and respond quickly to fluctuations in indole concentrations. However, as we now show, the function of indole has moved beyond a simple cue in many contexts and now plays a key role in regulating complex intra- and inter-specific interactions.

\section{Indole as an honest intraspecific signal}

The clearest evidence that indole functions as a signal comes from analyses of its role in mediating quorum-sensing (QS): the ability of microbial cells to measure population size and modulate their activities accordingly (Figure 2) [23]. QS systems 
are important for multicellular bacterial behavior such as sporulation, bioluminescence, and virulence factor production [24-26]. QS is used for sensing the same species, but it can also be used to sense populations of other bacteria [27], sometimes known as "eaves-dropping”. In the former case, indole is most likely being used as a signal, but in the latter it is more likely a cue (because the consensus is that it is unlikely that one bacterial species evolved a QS signal specifically to communicate with another bacterial species [23]).

The Escherichia coli volatile metabolic product indole is emerging as a signal that is important in QS interactions. Indole is produced by at least 27 different bacterial genera that produce tryptophanase (TnaA) [17], the enzyme that converts tryptophan into indole. Indole was first discovered as a signal in E. coli in which it activates gabT and $a s t D$ [28]. Using enterohemorrhagic E. coli (EHEC) and E. coli K12, it was then shown that indole is a QS signal [29] since it satisfies the four criteria for compounds to be called cell-to-cell signals [30]: (i) the putative signal must be produced during a specific stage (indole is produced primarily in the stationary-phase [28]), (ii) the putative signal must accumulate extra-cellularly and be recognized by a specific receptor (indole is a known extracellular signal $[28,31]$ that is exported by AcrEF [32] and is imported by Mtr [33] although it may pass through the membrane at a slower rate [34]), (iii) the putative signal must accumulate and generate a concerted response (indole has been shown to delay cell division [35]), and (iv) the putative signal must elicit a response that extends beyond the physiological changes required to metabolize or detoxify the signal (indole has been shown to control biofilms [17] and cell division [35,36] which are not related to indole metabolism). $E$. coli appears to have at least two QS systems when it lives in the mammalian gastrointestinal tract. At low temperatures, indole is the primary signal, while 
autoinducer 2 (AI-2) fills this role at higher temperatures [37].

\section{Indole as a manipulative interspecific bacterial signal}

Indole also mediates interactions with other species of bacterial cells. It reduces the pathogenicity of cells that do not synthesize it [38-40] and influences the biofilm formation of other cells [17]. For example, indole reduces the virulence of Pseudomonas aeruginosa in guinea pigs (Figure 3) by repressing the mexGHI-opmD multidrug efflux pump and the genes involved in the synthesis of pyocyanin $(p h z$ operon), 2-heptyl-3-hydroxy-4(1H)-quinolone (PQS) signal (pqs operon), pyochelin (pch operon) and pyoverdine ( $p v d$ operon) which results in reduced levels of pyocyanin, rhamnolipid, PQS and pyoverdine [38]. Each of these effects on other bacterial species is likely to be to the advantage of the signaler but to the detriment of the receiver. In this context, therefore, indole probably represents a form of coercion between species, mediated by manipulative signaling.

\section{Indole as an honest inter-kingdom bacterial signal with animals}

Remarkably, indole also mediates interactions between E. coli and the mammalian host in which it resides since it is one of the first compounds made by commensal bacteria in the mammalian gastrointestinal tract and has been shown to be beneficial by tightening gut epithelial cell junctions, thereby preventing invasion by pathogens $[41,42]$. Here we can consider indole to be an honest signal because the exclusion of pathogenic microbes is to the benefit of both the mammalian host and the commensal gut bacteria. Indole serves a similar defensive function in other animals that also have intimately associated microbiomes. For example, the indole derivative indole-3carboxaldehyde produced by microbes associated with the frog Smilisca phaeota [43] 
or the red-backed salamander, Plethodon cinereus [44], has been shown to repel or inhibit infection by fungal pathogens. These examples are slightly different to the honest signaling outlined above: they involve indole acting as an apparently manipulative signal to pathogenic fungi, to the detriment of their fitness, but to the benefit of the commensal microbes and their amphibian hosts.

Honest inter-kingdom signaling also occurs between microbes and animals in the context of dispersal. Here selection has resulted in close relationships between the one seeking the ride and the one providing it, and indole has been shown to play an important role in mediating the provision of this service. For example, the fetid fungus, Lysurus mokusin, relies upon the dispersal of its spores via fecal deposition of mycophagous insects. Insects are attracted to the scent of the fungus, of which indole is a key constituent, and feed upon the fungus. Consumed spores then pass through the insect alimentary tract, enhancing their ability to germinate and increasing their dispersal range [45]. Bioassays using a synthetic mixture of the characterized scent, which included about $7.5 \%$ indole (95-99\% pure), found that the odor of the fungus is attractive to the earwig, Anisolabis maritima (Bonelli) (Dermaptera: Anisolabididae) as well as flies belonging to 10 different genera from five families (Sarcophagidae, Calliphoridae, Muscidae, Sepsidae and Drosophilidae) [45]. Here indole is an important part of the honest signaling system that mediates dispersal.

However, not all instances of dispersal mediated by indole are so obviously part of an honest signaling system. For example, the Hippelates eye gnat (genus Tricimba (Lioy)) (Diptera: Chloropidae)) feeds on the mucous and sebaceous secretions around the eye of vertebrates and is capable of spreading microbial organisms that cause diseases, such as conjunctivitis (pink eye), anaplasmosis, and bovine mastitis in the vertebrate host, and which themselves produce indole during 
infections. Hwang et al. [46] found that gnats were especially attracted to odors that included indole or skatole - presumably because this environmental cue potentially guides them to a profitable feeding location. However, it is unknown whether gnats are more attracted to feeding locations infested with microbes, and unclear that these microbes produce indole for the purpose of attracting insects. Therefore we cannot conclude that indole is an honest inter-kingdom signal in this example.

The same problem exists for understanding dispersal of microbes mediated by the house fly Musca domestica L. (Diptera: Muscidae) and E. coli. In a study of chemical attractants to house flies, indole was determined to be a primary attractant [47]. When indole was compared with the closely related skatole or an indole-skatole mixture, it was determined that house flies respond more specifically to indole than skatole or the combination $[47,48]$. Indole is produced by E. coli, which is associated with vertebrate feces [49], which is an ephemeral resource, which lasts a few days or less depending on conditions [50]. As such, house flies must locate this resource quickly in order to maximize their use of it. Therefore, house flies utilize indole, which is a signature of feces and present in high concentrations, as a means to locate and colonize these resources. Adult flies attracted to the waste are then contaminated with E. coli and disperse it from this ephemeral resource to new locations [51]. Furthermore, adults that develop as larvae feeding on the manure are also contaminated with the bacteria and can disperse it into the surrounding areas [52], often resulting in the contamination of resources consumed by vertebrate hosts [53]. Nevertheless, it is unlikely that house flies and E. coli have evolved an honest indole signaling system to mediate $E$. coli dispersal. We should more conservatively conclude that flies are drawn to indole-rich resources because indole is a cue that conveys information about the value of the resource to the fly. Until further evidence 
is produced to indicate otherwise, the dispersal of $E$. coli is an incidental part of this process.

Similar processes of cue-mediated incidental dispersal probably also occur within the vertebrate carrion system where indole serves as a mediator of fly (Diptera) and beetle (Coleoptera) (Figure 4) attraction and utilization of associated resources. blow flies (Diptera: Calliphoridae) are attracted by indole to decomposing remains as a means to locate mates and provide resources to resulting offspring [54-57]. Research prior to Liu et al. [54] also determined that inhibiting behavioral responses by bacteria associated with carrion [58], specifically swarming by Proteus mirabilis, which is regulated by a quorum sensing pathway, resulted in reduced blow fly attraction and oviposition [59]. Furthermore, responses by flies to these bacteria were regulated by sex, age, and adult nutrition history [60]. As with the house fly example, resulting contaminated adults [61] disperse into the surrounding environment allowing for microbial colonization of other resources. Likewise, mosquitoes (Diptera: Culicidae) also utilize indole as a means to locate hosts for blood-meals [62] or oviposition sites [63]. However, any microbial dispersal that also ensues is likely to be a secondary part of their search for food and egg-laying sites.

\section{Indole as an honest inter-kingdom bacterial signal with plants}

Just as indole mediates interactions between microbes and animals, it similarly mediates interactions between bacteria and plants. Soil microbes are integral to plant health and influence root architecture [64]. In fact, over $80 \%$ of land plants are able to establish mutualistic interactions with soil microbes [65]. Signaling between soil microbes and plants, with indole serving as the medium through which this interaction occurs, has been well documented. Indole produced by microbes often stimulates 
plant growth directly [66] and in a dose-dependent manner, such as with the rhizobacterium Proteus vulgaris, whose production of indole accelerates cabbage and cress growth $[67,68]$. In many instances, the associated microbes release mineral nutrients to the plant and in return the plant releases carbon that is then utilized by the microbes [65].

Indole stimulates plant growth through the interplay of the auxin, cytokinin and brassinosteroid hormonal pathways [67]. When produced by soil bacteria, it is specifically able to promote early lateral root development by modulating the plant secondary root network via interference with the auxin-signaling machinery, an essential local signal for lateral root growth [66,69]. Indole-3-acetic acid (IAA) is among the most common natural auxin growth regulators found in plants and exerts a positive influence on root growth and length, thus increasing the total root surface area [70]. However, as mentioned above, a dose-dependent, plant-growth-promoting property exists and long-term exposure to high concentrations of indole can have negative effects on growth and development, as demonstrated with the rockcress, Arabidopsis thaliana (L.) [69]. The bacterium Pseudomonas putida and the fungus Trichoderma atrovirid both produce IAA, which can increase the weight of the shoots and roots of tomato plant seedlings, but these microbes were also determined to have the capacity to reduce the deleterious effect of excess IAA by microbial degradation [71]. Therefore, microbes may help plants in two ways: by stimulating growth through production of an indole derivative and by helping to degrade harmful excesses in concentrations of these indole-related compounds. Whether these compounds are functioning as cues or signals in this context remains to be formally determined. However, it is conceivable that indole is an honest signal in this context because the microbes potentially benefit by closely regulating plant growth to 
optimize the levels of carbon the plant then releases back to them.

\section{Indole signaling between plant cells}

Indole also plays an important role in regulating plant defense mechanisms. In the case of rice Oryza sativa, GH3-8, an auxin-responsive gene responds to indole-3acetic acid and activates disease resistance via jasmonic acid and salicyclic acid signaling-independent pathways [72]. Here this variant of indole serves a signaling function within the plant, to coordinate its defense mechanisms. In addition, following attack by herbivores, plants can release a suite of volatile organic compounds, including indole, which can induce nearby plants of the same species to enhance their defensive mechanisms [73]. This phenomenon, called priming, triggers increased transcription of defense-related genes thus allowing nearby plants to respond more rapidly and robustly to an imminent assault [74]. For example, in a study by Erb et al. [75], maize was injured and treated with African cotton leafworm, Spodoptera littoralis Boisduval (Lepidoptera: Nocuidae) regurgitate. The authors found that indole was produced within 45 min and peaked after about $2 \mathrm{~h}$. They also determined that in the presence of indole the production of volatiles and terpenoids by plants that were subsequently injured was enhanced as a result of priming. The simplest interpretation here is that plants are eavesdropping on indole cues produced by their neighbors, to adaptively modulate their defense mechanisms against the likelihood of herbivore attack. However, it is possible that indole is used as a signal in this context if neighboring plants are closely related, or if collectively unrelated neighboring plants can more effectively repel attack by herbivores. If these conditions are met, then the indole-producing plant gains fitness benefits by producing indole, either by helping to defend relatives against attack or by reducing its own future vulnerability 
to attack by herbivores.

\section{Indole in manipulative inter-kingdom signaling by plants}

Indole production by some plants directly prevents fungal infection. For example, indole produced by barley, Hordeum vulgare L. cv. Goseshikoku and cv. Morex can reduce the likelihood of powdery mildew, Blumeria graminis f.sp. hordei, infection [76]. Similarly, rice, Sekiguchi lesion (sl)-mutant produces indole to reduce rice blast fungal infection [77], while indole and other associated compounds also reduce the likelihood of infection by the fungus responsible for brassica dark leaf spot, Alternaria brassicicola [78]. Here indole can be viewed as a manipulative signal, just as it is when used by microbes carried by amphibian as a defense against fungi [43]: the plant gains fitness from indole production, while the fungi lose fitness. Manipulative signaling also apparently occurs between the mouse-ear, Arabidopsis thaliana, and the cabbage white butterfly [79]. Here some concentrations of indole produced by the plant inhibit oviposition by the butterfly, although at other doses, oviposition is enhanced. The dose-dependent response to indole by the butterfly might explain why manipulative signaling by the plant can persist in this context and the butterfly has not evolved to ignore it: any fitness costs to the butterfly through lost fecundity are potentially offset by fitness it might gain in response to other levels of indole signaling.

\section{Indole in honest inter-kingdom signaling by plants}

The cocktail of chemicals released by plants in response to damage by herbivores has also been implicated in inter-kingdom signaling, with indole serving a key role in this function. For example, the release of indole directly attracts parasitoids, which then 
kill the plant's insect herbivores. An example comes from Alborn et al. [80] and involves the beet armyworm caterpillar Spodoptera exigua (Hübner) (Lepidoptera: Noctuidae). This caterpillar secretes volicitin (a fatty acid derivative regurgitate $\mathrm{N}$ (17-hydroxylinolenoyl)-L-glutamine) while consuming plants, such as maize, Zea mays (L.). Contact between volicitin and the plant elicits the release of a blend of volatile terpenoids and indole systemically from the maize plant, not just from the damaged maize leaves. Volicitin selectively activates the formation of free indole [81], drawing in parasitoids which lay their eggs in the caterpillar. Here indole is an honest signal because it enables both the plant and the parasitoid to gain fitness benefits.

In other cases, indole serves a more indirect function in recruiting natural enemies of the arthropod herbivore. The volatiles produced by plants subsequent to herbivore feeding are complex blends of compounds resulting from three primary biosynthetic pathways: the terpenoid, the shikimate, and the fatty acid degradation pathway [82]. Indole produced from the shikimic acid pathway can play a role in indirect defense because it facilitates the release of a different volatile signal from a damaged plant that attracts natural enemies of the arthropod herbivore inflicting the damage [83]. Here, indole's function is merely to mediate communication within the plant, which in turn leads to the release of a second honest signal that is received by the animal.

\section{Indole in mating displays by animals}

Indole has been shown to be involved in pheromonal displays that are used for mate attraction in animals. For the most part, these are displays by females for attracting males. For example, males of the scarab beetle, Holotrichia reynaudi Hope 
(Coleoptera: Scarabaeidae) rely on a mixture of abdominal exudates, including indole, produced by the female to locate a partner [84]. However, in the dung beetle, Kheper bonelli (MacLeay) (Coleoptera: Scarabaeidae) males produce and release a proteinaceous secretion to attract females for mating. Within this proteinaceous carrier material are putative sex pheromones, among which indole was identified [85]. Given that this particular species relies on dung, which typically contains high levels of E. coli (which produces indole as previously mentioned), it would be interesting to determine whether indole production is truly by the insect or by the E. coli harbored within the insect. In general, although indole is present in these pheromonal cocktails, its function in luring a mate remains unclear. As is illustrated by the examples we consider next, it might function to convey important information to a potential partner, or it may simply amplify information conveyed by other compounds in the pheromone. It might even play no role at all in mate attraction. More work is required to distinguish these different possibilities.

\section{The function of indole in complex displays: information carrier or}

\section{amplifier?}

In many of the examples discussed above, indole is part of a complex cocktail of volatiles emitted by a signaler. To understand its specific function in these contexts, we must turn to evolutionary theory connected with complex, or multicomponent displays (summarized above). One suggestion here is that some elements of a complex display serve to amplify other parts of the display [11]. Indole seems to serve exactly this function in the signaling that takes place between the gourd family of flowering plants (Cucurbitaciae) and diabroticite rootworm beetles (Coleoptera: Chrysomelidae: Luperini). These organisms are anciently associated with one another 
and have likely coevolved through their associated chemical ecology $[86,87]$. The Cucurbita blossom is a source of nectar and pollen for diabroticite beetles, which are attracted by its odorous bouquet, and which includes indole as a volatile [88]. Most Cucurbitaciae produce a secondary compound called cucurbitacin, a triterpene hydrocarbon containing an indole structure [89]. Cucurbitacins are bitter and often toxic semiochemicals that serve to protect the plants from attack by invertebrate and vertebrate herbivores. Diabroticite beetles, however, use these compounds as kairomones for locating the blossom. They are able to feed on the cucurbits and store the bitter cucurbitacins in their blood and tissues as allomones to deter predation [87].

The role of indole in attracting the beetles to the blossom is to act as an amplifier. The diabroticite beetles, Acalvmma vitlatum, Diabrotica u. howardi, D. virgifera virgifera, and D. barberi, are only weakly to moderately attracted to indole as a single compound [90]. However, when combined with other olfactants from Cucurbita blossoms, indole increased olfactory responses in diabroticite beetles synergistically by 2 to 4 fold $[88,90]$. Further evidence that indole amplifies the attraction of other volatiles to these beetles comes from experiments using insect traps for diabroticite rootworm beetles, in bean, Phaseolus vulgaris, and soybean, Glycine $\max$, fields. Traps baited with veratrole + indole + phenylacetaldehyde caught 6.5 and 3.5 times more beetles than solvent controls in soybean and common bean plots, respectively; traps baited with 1,2,4-trimethoxybenzene + indole + trans-cinnamaldehyde) caught 6.7 and 3.5 times more beetles, respectively [91]. Thus indole has evolved to be part of the Cucurbita blossom's odiferous display seemingly because it amplifies the response by beetles to other volatiles in the bouquet.

Indole might serve a similar amplifying function in the scent profile of other plant species, as demonstrated in a study by Friberg et al. [92]. Here it was found to 
be one of the compounds within the unique floral profiles produced by two different woodstar plants, Lithophragma bolanderi and L. cymbalaria (Saxifragaceae), which attract the parasitic moth, Greya politella (Walsingham) (Prodoxidae), for pollination. Female moths responded most strongly to the uniquely distinctive scents from their local host species and were thereby more likely to pollinate the local plants. It would be interesting in future work to determine whether this divergent response to floral scents has been facilitated by indole.

In other floral scents, however, indole might be used by the plant as a signal to manipulate insects into providing a pollination service. For example, the composition of the floral scent of the sapromyiophilous, Periploca laevigata, was investigated because of its ability to lure in the common house fly as a pollinator species [93]. The most abundant compound identified in the scent disseminated from cultivated sapromyiophilous was indole (39\%), which attracted both male and female flies [93]. Presumably the flies use indole as a cue for locating oviposition or food resources and the plant has evolved a manipulative signal, in which the insects are sensorily trapped into visiting the plant and pollinate it in the process.

\section{Conclusions}

In this review we have attempted to explain why indole is used so ubiquitously in nature, and how it functions to modulate interactions among diverse organisms. We suggest that indole is used so widely, and by such different organisms, because it derives from the metabolism of tryptophan, a resource that is essential for many species yet rare in nature. These properties make it a valuable, environmental cue for resources that are almost universally important for promoting fitness. By surveying a broad literature, we find that indole is used to coordinate actions within organisms, to 
influence the behavior of conspecifics and can even be used to change the behavior of species that belong to other kingdoms. This is variously achieved by indole acting as a cue, a manipulative signal, and an honest signal, as well as an amplifier for information conveyed by other molecules. Importantly, these distinct functions of indole transcend different kingdoms of organisms. These roles across kingdoms for indole make it special to the extent it is widely used but not necessarily unique; for example, the bacterial QS signal $N$-acyl-1-homoserine lactone from the opportunistic pathogen Pseudomonas aeruginosa represses the mammalian innate immune system [94]

We suggest that future work could profitably build on the conclusions of this article by using indole in interventions to manage crop pests and to control vectors of pathogens. Existing biology reviewed here suggests that such insect species could be surprisingly vulnerable to being manipulated in this way. Finally, we wonder whether indole could even be deployed to promote the pollination services provided by insects of economically important crop plants, since existing evidence suggests this might enhance the attractiveness of the plant to potential pollinators, possibly thereby boosting pollination rates. 
Figure 1. Mechanisms for the physiological actions of indole in animals. In animals, indole can scavenge free radical species and exert anti-oxidative effects [95], and can also enhance expression of xenobiotic-metabolizing enzymes (e.g., cytochrome P450) and immune response through binding to aryl hydrocarbon receptors (ligand-activated transcription factors) [96]. These actions of indole result in the amelioration of oxidative stress (such as UV radiation- or oxidant-induced DNA damage). Through binding to the serotonin receptor and serving as an $\alpha_{1 A^{-}}$ adrenoceptor antagonist [97], indole modulates animal behavior, the contraction of smooth muscle, gut motility, and food intake [98]. By interacting with iron in hemecontaining oxygenases [95], indole plays a role in whole-body aerobic metabolism. Indole also regulates the release of secretion of luteinizing hormone, and, therefore male and female reproduction [99]. Finally, indole affects the metabolism and activity of gut microbes, thereby sustaining intestinal health $[100,101]$.

Figure 2. The hypothesized function of quorum sensing. Bacterial cells produce signal molecules, which can be used as a source of information about the density of cells in their environment. It has been shown that cells use this information to control the expression of density dependent traits, such as protease production. On the left, the diagram shows how beneficial exo-products can be easily lost, providing little or no benefit to cells, the right hand side shows how exo-products are more likely to benefit surrounding cells at high densities. [102].

Figure 3. Reduction of virulence of $P$. aeruginosa in guinea pigs by 7hydroxyindole (7HI). Colonization and clearance of $P$. aeruginosa PAO1 pre-treated with 7HI or solvent (DMF) prior to infection of guinea pigs by aerosol with $\sim 2 \times 10^{5}$ cfu. Average of five replicates, and one standard deviation is shown (A). Real-time analysis of $P$. aeruginosa PAO1 pre-treated with 7HI or solvent (DMF) in the acute guinea pig infection model (representative guinea pigs are shown for each group and are imaged laterally) using the Xenogen IVIS CCD camera (B). Color bar represents the intensity of luminescent signal in photons $/ \mathrm{sec} / \mathrm{cm}^{2}$ from low (blue) to high (red) [38].

Figure 4. Arthropods commonly colonizing vertebrate carrion. (a) Beetles (e.g. Nicrophorus vespilloides [Coleoptera: Silphidae], photo: Tom Houslay) and (b) flies (e.g. Chrysomya rufifacies and Cochliomyia macellaria [Diptera: Calliphoridae], photo: C.C. Heo) are the primary invertebrate consumers of vertebrate carrion. Such invertebrates use indole and other volatiles to locate such resources essential for mate location as well as adult and larval nutrition. 


\section{References}

1 Ezenwa Vo, Gerardo NM, Inouye DW, Medina M, et al. 2012. Animal behavior and the microbiome. Science 338: 198-9.

2 Ezenwa Vo, Williams AE. 2014. Microbes and animal olfactory communication: Where do we go from here? Bioessays 36: 847-54.

3 Davis TS, Crippen TL, Hofstetter RW, Tomberlin JK. 2013. Microbial volatile emissions as insect semiochemicals. J Chem Ecol 39: 840-59.

4 Lee J-H, Wood TK, Lee J. 2015. Roles of indole as an interspecies and interkingdom signaling molecule. Trends Microbiol 23: 707-18.

5 Taber DF, Tirunahari PK. 2011. Indole synthesis: A review and proposed classification. Tetrahedron 67: 7195-210.

6 Bradbury J, Vehrencamp S. 1998. Principles of Animal Communication: MA: Sinauer Press.

7 Davies NB, Kilner RM, Noble DG. 1998. Nestling cuckoos, Cuculus canorus, exploit hosts with begging calls that mimic a brood. Proc Royal Soc B: Biol Sci 265: 673-8.

8 Dawkins R. 1976. The Selfish Gene Oxford: Oxford University Press.

9 Rowe C. 1999. Receiver psychology and the evolution of multicomponent signals. Ani Behav 58: 921-31.

10 Johnstone RA. 1996. Multiple displays in animal communication: 'Backup signals' and multiple messages'. Proc Royal Soc B: Biol Sci 351: 329-38.

11 Hasson 0. 1989. Amplifiers and the handicap principle in sexual selection- a different emphasis. Proc Royal Soc B: Biol Sci 235: 383-406.

12 Kilner RM, Noble DG, Davies NB. 1999. Signals of need in parentoffspring communication and their exploitation by the cuckoo. Nature 397: 667-72.

13 Kilner RM. 2002. The Evolution of Complex Begging Displays. In Wright J, Leonard ML, eds; The Evolution of Begging: Competition, Cooperation and Communication. Dordrecht: Springer Netherlands. p 87-106.

14 Wu G. 2013. Amino Acids: Biochemistry and Nutrition: Boca Raton, FL: CRC Press.

15 Yao K, Yin KL, Feng ZM, Tang ZR, et al. 2011. Tryptophan metabolism in animals: important roles in nutrition and health. Front Biosci S3: 286-97.

16 Kundu P, Pettersson S. 2014. Immunology: Mammalian watchdog targets bacteria. Nature 512: 377-8.

17 Lee J, Jayaraman A, Wood TK. 2007. Indole is an inter-species biofilm signal mediated by SdiA. BMC Microbiol 7: 42.

18 Tsavkelova EA, Cherdyntseva TA, Klimova SY, Shestakov AI, et al. 2007. Orchid-associated bacteria produce indole-3-acetic acid, promote seed germination, and increase their microbial yield in response to exogenous auxin. Arch Microbiol 188: 655-64.

19 De-Bashan LE, Antoun H, Bashan Y. 2008. Involvement of indole-3acetic acid produced by the growth-promoting bacterium Azospirillum spp. in promoting growth of Chlorella vulgaris. J Phycol 44: 938-47.

20 Amin SA, Hmelo LR, van Tol HM, Durham BP, et al. 2015. Interaction and signalling between a cosmopolitan phytoplankton and associated bacteria. Nature 522: 98-101. 
21 Kulkarni GB, Sajjan SS, Karegoudar TB. 2011. Pathogenicity of indole3-acetic acid producing fungus Fusarium delphinoides strain GPK towards chickpea and pigeon pea. Eur J Plant Pathol 131: 355-69.

22 Khan AR, Ullah I, Waqas M, Shahzad R, et al. 2015. Plant growthpromoting potential of endophytic fungi isolated from Solanum nigrum leaves. World J Microbiol Biotechnol 31: 1461-6.

23 Diggle SP, Gardner A, West SA, Griffin AS. 2007. Evoultionary theory of quorum sensing: When is a signal not a signal? Proc Royal Soc B: Biol Sci 362: 1241-9.

24 Eberl L, Winson MK, Sternberg C, Stewart GSAB, et al. 1996. Involvement of N-acyl-l-homoserine lactone autoinducers in controlling the multicellular behaviour of Serratia liquefaciens. Mol Microbiol 20: 12736.

25 Kolter R, Losick R. 1998. One for all and all for one. Science 280: 226-7.

26 Salmond GPC, Bycroft BW, Stewart GSAB, Williams P. 1995. The bacterial 'enigma': Cracking the code of cell-cell communication. Mol Microbiol 16: 615-24.

27 Xavier KB, Bassler BL. 2005. Interference with AI-2-mediated bacterial cell-cell communication. Nature 437: 750-3.

28 Wang D, Ding X, Rather PN. 2001. Indole can act as an extracellular signal in Escherichia coli.J Bacteriol 183: 4210-6.

29 Lee J, Bansal T, Jayaraman A, Bentley WE, et al. 2007. Enterohemorrhagic Escherichia coli biofilms are inhibited by 7hydroxyindole and stimulated by isatin. Appl Environ Microbiol 73: 41009.

30 Winzer $\mathbf{K}$, Hardie KR, Williams P. 2002. Bacterial cell-to-cell communication: sorry, can't talk now - gone to lunch! Curr Opin Microbiol 5: 216-22.

31 Hirakawa H, Inazumi Y, Masaki T, Hirata T, et al. 2005. Indole induces the expression of multidrug exporter genes in Escherichia coli. Mol Microbiol 55: 1113-26.

32 Kawamura-Sato K, Shibayama K, Horii T, Iimuma Y, et al. 1999. Role of multiple efflux pumps in Escherichia coli in indole expulsion. FEMS Microbiol Lett 179: 345-52.

33 Yanofsky C, Horn V, Gollnick P. 1991. Physiological studies of tryptophan transport and tryptophanase operon induction in Escherichia coli. J Bacteriol 173: 6009-17.

34 Vega NM, Allison KR, Khalil AS, Collins JJ. 2012. Signaling-mediated bacterial persister formation. Nat Chem Biol 8: 431-3.

35 Chant EL, Summers DK. 2007. Indole signalling contributes to the stable maintenance of Escherichia coli multicopy plasmids. Mol Microbiol 63: 3543.

36 Chimerel C, Field CM, Pinero-Fernandez S, Keyser UF, et al. 2012. Indole prevents Escherichia coli cell division by modulating membrane potential. Biochim Biophys Acta 1818: 1590-4.

37 Lee J, Zhang X-S, Hegde M, Bentley WE, et al. 2008. Indole cell signaling occurs primarily at low temperatures in Escherichia coli. ISME J 2: 100723. 
38 Lee J, Attila C, Cirillo SL, Cirillo JD, et al. 2009. Indole and 7hydroxyindole diminish Pseudomonas aeruginosa virulence. Microb Biotechnol 2: 75-90.

39 Chu W, Zere TR, Weber MM, Wood TK, et al. 2012. Indole production promotes Escherichia coli mixed-culture growth with Pseudomonas aeruginosa by inhibiting quorum signaling. Appl Environl Microbiol 78: 411-9.

40 Hidalgo-Romano B, Gollihar J, Brown SA, Whiteley M, et al. 2014. Indole inhibition of $\mathrm{N}$-acylated homoserine lactone-mediated quorum signalling is widespread in Gram-negative bacteria. Microbiol 160: 246473.

41 Bansal T, Alaniz RC, Wood TK, Jayaraman A. 2010. The bacterial signal indole increases epithelial-cell tight-junction resistance and attenuates indicators of inflammation. Proc Nat Acad of Scis 107: 228-33.

42 Shimada Y, Kinoshita M, Harada K, Mizutani M, et al. 2013. Commensal bacteria-dependent indole production enhances epithelial barrier function in the colon. PLOS ONE 8: e80604.

43 Lam BA, Walton DB, Harris RN. 2011. Motile zoospores of Batrachochytrium dendrobatidis move away from antifungal metabolites produced by amphibian skin bacteria. EcoHealth 8: 36-45.

44 Brucker RM, Harris RN, Schwantes CR, Gallaher TN, et al. 2008. Amphibian chemical defense: Antifungal metabolites of the microsymbiont Janthinobacterium lividum on the salamander Plethodon cinereus. J Chem Ecol 34: 1422-9.

45 Chen G, Zhang RR, Liu Y, Sun WB. 2014. Spore dispersal of fetid Lysurus mokusin by feces of mycophagous insects. J Chem Ecol 40: 893-9.

46 Hwang Y-S, Mulla MS, Axelrod H. 1976. Attractants for synanthropic flies. Identification of attractants and coattractants for Hippelates eye gnats (Diptera, Chloropidae). J Agr Food Chem 24: 164-9.

47 Mulla MS, Hwang YS, Axelrod H. 1977. Attractants for synanthropic flies: Chemical attractants for domestic flies. J Econ Entomol 70: 644-8.

48 Mulla M, Ridsdill-Smith J. 1986. Chemical attractants tested against the Australian bush fly Musca vetustissima (Diptera: Muscidae). J Chem Ecol 12: 261-70.

49 Alam MJ, Zurek L. 2004. Association of Escherichia coli 0157:H7 with houseflies on a cattle farm. Appl Environ Microbiol 70: 7578-80.

50 Mohr C0. 1943. Cattle dropping as ecological units. Ecol Mon 13: 275-98.

51 Zurek L, Ghosh A. 2014. Insects represent a link between food animal farms and the urban environment for antibiotic resistance traits. Appl Environ Microbiol 80: 3562-7.

52 Rochon K, Lysyk TJ, Selinger LB. 2004. Persistence of Escherichia coli in immature house fly and stable fly (Diptera: Muscidae) in relation to larval growth and survival. J Med Entomol 41: 1082-9.

53 Wasala L, Talley JL, Desilva U, Fletcher J, et al. 2013. Transfer of Escherichia coli 0157:H7 to spinach by house flies, Musca domestica (Diptera: Muscidae). Phytopathol 103: 373-80.

54 Liu W, Longnecker M, Tarone AM, Tomberlin JK. 2016. Response of Lucilia sericata (Diptera: Caliphoridae) to compounds from microbial decomposition of larval resources. Ani Behav 115: 217-25. 
55 Frederickx C, Dekeirsschieter J, Verheggen FJ, Haubruge E. 2012. Responses of Lucilia sericata Meigen (Diptera: Calliphoridae) to cadaveric volatile organic compounds. J Forensic Sci 57: 386-90.

56 Brodie BS, Babcock T, Gries R, Benn A, et al. 2016. Acquired smell? Mature females of the common green bottle fly shift semiochemical preferences from feces feeding sites to carrion oviposition sites. J Chem Ecol 42: 40-50.

57 Dethier VG. 1947. Chemical Insect Attractants and Repellents Philadelphia, PA: The Blakiston Company.

58 Barnes KM, Gennard DE, Dixon RA. 2010. An assessment of the antibacterial activity in larval excretion/secretion of four species of insects recorded in association with corpses, using Lucilia sericata Meigen as the marker species. Bull Entomol Res 100: 635-40.

59 Ma Q, Fonseca A, Liu W, Fields AT, et al. 2012. Proteus mirabilis interkingdom swarming signals attract blow flies. ISME J 6: 1356-66.

60 Tomberlin JK, Crippen TL, Tarone AM, Singh B, et al. 2012. Interkingdom response of flies to bacteria mediated by fly physiology and bacterial quorum sensing. Ani Behav 84: 1449-56.

61 Singh B, Crippen TL, Zheng L, Fields AT, et al. 2015. A metagenomic assessment of the bacteria associated with Lucilia sericata and Lucilia cuprina (Diptera: Calliphoridae). Appl Microbiol Biot 99: 869-83.

62 Meijerink J, Braks MAH, Brack AA, Adam W, et al. 2000. Identification of olfactory stimulants for Anopheles gambiae from human sweat samples. J Chem Ecol 26: 1367-82.

63 Bohbot JD, Jones PL, Wang G, Pitts RJ, et al. 2011. Conservation of indole responsive odorant receptors in mosquitoes reveals an ancient olfactory trait. Chem Senses 36: 149-60.

64 Ortiz-Castro R, Contreras-Cornejo H, Macias-Rodriguez L, LopezBucio J. 2009. The role of microbial signals in plant growth and development. Plant Sig Behav 4: 701-12.

65 Morgan JAW, Bending GD, White PJ. 2005. Biological costs and benefits to plant-microbe interactions in the rhizosphere. J Exp Bot 56: 1729 - 39.

66 Bailly A, Groenhagen U, Schulz S, Geisler M, et al. 2014. The interkingdom volatile signal indole promotes root development by interfering with auxin signalling. The Plant J 80: 758-71.

67 Bhattacharyya D, Garladinne M, Lee Y. 2015. Volatile indole produced by rhizobacterium Proteus vulgaris JBLS202 stimulates growth of Arabidopsis thaliana through auxin, cytokinin and brassinosteroid patyhways. J Plant Growth Reg 34: 158-68.

68 Yu S, Lee Y. 2013. Plant growth promotin rhizobacterium Proteus vulgaris JBLS202 stimulates seedling growth of Chinese cabbage through indole emission. Plant Soil 370: 485-95.

69 Blom D, Fabbri C, Connor EC, Schiestl FP, et al. 2011. Production of plant growth modulating volatiles is widespread among rhizosphere bacteria and strongly depends on culture conditions. Environ Microbiol 13: 3047-58.

70 Vessey JK. 2003. Plant growth promoting rhizobacteria as biofertilizers. Plant and Soil 255: 571-86. 
71 Gravel V, Antoun H, Tweddell RJ. 2007. Growth stimulation and fruit yield improvement of greenhouse tomato plants by inoculation with Pseudomonas putida or Trichoderma atroviride: Possible role of indole acetic acid (IAA). Soil Biol Biochem 39: 1968-77.

72 Ding X, Cao Y, Huang L, Zhao J, et al. 2008. Activation of the indole-3acetic acid-amido synthetase GH3-8 suppresses expansin expression and promotes salicylate- and jasmonate-independent basal immunity in rice. The Plant Cell 20: 228-40.

73 Kessler A, Halitschke R, Diezel C, Baldwin IT. 2006. Priming of plant defense responses in nature by airborne signaling between Artemisia tridentata and Nicotiana attenuata. Oecologia 148: 280-92.

74 Baldwin IT, Halitschke R, Paschold A, von Dahl CC, et al. 2006. Volatile signaling in plant-plant interactions: "talking trees" in the genomics era. Science 311: 812-5.

75 Erb M, Veyrat N, Robert C, Xu H, et al. 2015. Indole is an essential herbivore-induced volatile priming signal in maize. Nature Comm 6: 6273.

76 Matsuo H, Taniguchi K, Hiramoto T, Yamada T, et al. 2001. Gramine increase associated with rapid and transient systemic resistance in barley seedlings induced by mechanical and biological stresses. Plant Cell Physiol 42: 1103-11.

77 Ueno M, Kihara J, Honda Y, Arase S. 2005. Effects of some indole-related comopunds on the infection behavior of Magnaporthe grisea. J Gen Plant Pathol 71: 196-9.

78 Buxdorf K, Yaffe H, Barda O, Levy M. 2013. The effects of glucosinolates and their breakdown products on necrotrophic fungi. PLOS ONE 8: e70771.

79 de Vos M, Kriksunov KL, Jander G. 2008. Indole-3-acetonitrile production from indole glucosinolates deters oviposition by Pieris rapae. Plant Physiol 146: 916-26.

80 Alborn HT, Turlings TCJ, Jones TH, Stenhagen G, et al. 1997. An elicitor of plant volatiles from beet armyworm oral secretion. Sci Cul 276: 945-79.

81 Frey M, Stettner C, Pare PW, Schmelz EA, et al. 2000. A herbivore elicitor activates the gene for indole emission in maize. Proc Natl Acad Sci 97: 14801-6.

82 Dudareva N, Negre F, Nagegowda D, Orlova I. 2006. Plant volatiles: Recent advances and future perspectives. Critical Rev Plant Sci 25: 41740.

83 Degenhardt J. 2009. Indirect defense responses to herbivory in grasses. Plant Physiol 149: 96-102.

84 Ward A, Moore C, Anitha V, Wightman J, et al. 2002. Identification of the sex pheromone of Holotrichia reynaudi.J Chem Ecol 28: 515-22.

85 Burger B, Petersen W, Ewig B, Neuhaus J, et al. 2008. Semiochemicals of the Scarabaeinae VIII. Identification of active constituents of the abdominal sex-attracting secretion of the male dung beetle, Kheper bonellii, using gas chromatography with flame ionization and electroantennographic detection in parallel. J Chrom A 1186: 245-53.

86 Metcalf R, L., Lampman RL. 1989. The chemical ecology of Diabroticites and Cucurbitaceae. Experientia 45: 240-7. 
87 Metcalf RU. 1986. Coevolutionary adaptations of rootworm beetles (Coleptera: Chrysomelidae) to cucurbitacins. J Chem Ecol 12: 1109-24.

88 Anderson JF, Metcalf RL. 1986. Identification of a volatile attractant for Diabrotica and Acalynnna spp. from blossoms of Cucurbita maxima Duchesne. J Chem Ecol 12: 687-99.

89 Metcalf RL, Metcalf RA, Rhodes AM. 1980. Cucurbitacins as kairomones for diabroticite beetles. Proc Nat Acad Sci 77: 3769-72.

90 Metcalf R, Lampman R, Deem-Dickson L. 1995. Indole as an olfactory synergist for volatile kairomones for diabroticite beetles. J Chem Ecol 21: 1149-62.

91 Ventura MU, Martins MC, Pasini A. 2000. Responses of Diabrotica speciosa and Cerotoma arcuata tingomariana (Coleoptera: Chrysomelidae) to volatile attractants. Flor Entomol 83: 403-10.

92 Friberg M, Schwind C, Roark LC, Raguso RA, et al. 2014. Floral scent contributes to interaction specificity in coevolving plants and their insect pollinators. J Chem Ecol 40: 955-65.

93 Zito P, Dotterl S, Sajeva M. 2015. Floral volatiles in a sapromyiophilous plant and their importance in attracting house fly pollinators. J Chem Ecol 41: 340-9.

94 Kravchenko VV, Kaufmann GF, Mathison JC, Scott DA, et al. 2008. Modulation of gene expression via disruption of NF-kappaB signaling by a bacterial small molecule. Science 321: 259-63.

95 Modelli A, Jones D, Pshenichnyuk SA. 2013. Electron attachment to indole and related molecules. J Chem Phys 139: 184305.

96 Fujii-Kuriyama Y, Mimura J. 2005. Molecular mechanisms of AhR functions in the regulation of cytochrome P450 genes. Biochem Biophys Res Commun 338: 311-7.

97 Xu W, Huangg JJ, Shao BH, Xu XJ, et al. 2015. X-ray crystallography, DFT calculations and molecular docking of indole-arylpiperazine derivatives as $\alpha 1 \mathrm{~A}$-adrenoceptor antagonists. Molecules 20: 19674-89.

98 Wu G. 2009. Amino acids: metabolism, functions, and nutrition. Amino Acids 37: 1-17.

99 Dai Z, Wu Z, Hang S, Zhu W, et al. 2015. Amino acid metabolism in intestinal bacteria and its potential implications for mammalian reproduction. Mol Hum Reprod 21: 389-409.

100 Dai ZL, Wu G, Zhu WY. 2011. Amino acid metabolism in intestinal bacteria: links between gut ecology and host health. Fron Biosci (Landmark edition) 16: 1768-86.

101 Wang H, Ji Y, Wu G, Sun KJ, et al. 2015. L-Tryptophan activates mammalian target of rapamycin and enhances expression of tight junction proteins in intestinal porcine epithelial cells. J Nutr 145: 115662.

102 Davies NB, Krebs JR, West SA. 2012. An Introduction to Behavioural Ecology: John Wiley \& Sons, Ltd.

103 Zúniga GE, Salgado MS, Corcuera LJ. 1985. Role of an indole alkaloid in the resistance of barley seedlings to aphids. Phytochem 24: 945-7.

104 Bansal T, Englert D, Lee J, Hegde M, et al. 2007. Differential effects of epinephrine, norepinephrine, and indole on Escherichia coli 0157:H7 
chemotaxis, colonization, and gene expression. Infect Immun 75: 4597607. 